\title{
A Case Report of the At-Home Application of Maggot in Treatment of Infected Wound in A Diabetic Patient
}

\section{Abstract}

Use of sterile maggot larvae in the removal of infected tissue has been practiced for centuries in different cultures. The practice was declined once antibiotics were introduced. Today, due to antibiotic resistant development or when conventional treatment is not possible, use of maggot therapy in management of infection in different conditions has been shown to be effective. Maggot therapy has been reported to be especially effective in treating diabetic wounds once it starts early in the process.

Here, we are reporting a case study where maggot therapy was applied by a family member at home to an elderly patient when two months treatment of a wound in a diabetic foot with conventional antibiotics showed no improvement in progression of healing. However, addition of maggot therapy at home was effective in complete healing of the wound in three months. Our observations indicate a decrease in exudation and edema a week after the application of maggot therapy, significant improvement three weeks after, and a complete healing after three months of maggot therapy.

Based on these observations we are concluding that maggot therapy can be safely applied by an untrained person at home setting. This is a low cost and convenient alternative to antibiotic treatment that can be used at home for treating diabetic wounds without the need of a professional.

Keywords: Maggot Therapy; Diabetes; Infection

\section{Introduction}

According to 2014 National Diabetes Statistics Report and 2016 WHO report, currently 29.1 million (9.3\%) of Americans and 422 million of world population are living with diabetes [1] and the numbers are increasing which is estimated to increase by two fold by 2030 [2]. One of the complications of diabetes is a slow rate of wound healing. A poorly functioning immune system in these patients increases the risk for developing an infection in wounded area and makes the treatment a challenging task. In many cases when an infection develops, treatment with conventional antibiotic is not very successful. Untreated infection can increase the risk of developing gangrene, sepsis or osteomyelitis and contributes to number one reason for limb amputation in these patients.

Maggot therapy, also known as maggot debridement therapy (MDT) and larvae therapy, is a method of using a germ-free, safe and effective species of maggot to remove infected tissue. MDT has been used in many cultures for the treatment of infections and the practice goes back to ancient time [3]. MDT was replaced with antibiotics once they became available in 1928. Due to the development of antibiotic resistant in some patients, there has been an increased interest for the use of MDT as an alternative treatment of infected wounds. Here we are reporting the use of MDT in Iran for treating an infected wound in a diabetic patient where antibiotic resistant was developed.

\section{Case Presentation}

An 82 years old male patient with diabetes for more than 40 years developed infected wounds on lower part of leg (top of lateral malleolus) due to improper casting of the leg after a fall in March of 2016. Treatment with different antibiotics for more than two months proved to be unsuccessful and at-home selftreatment with maggot was chosen by the patient and his family.

\section{Materials and Methods}

This was as observational study and an informed consent was signed by the patient before the start of observation to permit photography during the observation and for future publication of the observations.

The germ-free larvae of the Phaenicia (=Lucilia) sericata (Calliphoridea; Diptera) were used to treat and manage the wound. The larvae were purchased from established colonies in the insectory of 'Avan Knowledge for Life, Science and Technology of Khozestown Park', Khozestown, Iran.

\section{Treatment}

Maggots were prepared as sterile maggot-impregnated gauze (Biobag). Common recommended treatment with maggots is 3 days. In cases where there is an extensive infection, the treatment with a fresh batch of maggots can be repeated. In the present case, at the start of treatment with maggots, after cleaning the wound 
with normal saline, maggots were applied to the wound topped with a layer of light gauze and secured in place with adhesive tapes. The outer gauze was changed after 24 hours and Biobag, which at this point had released all the larvae, was discarded. The outer gauze was changed once or twice daily for the next 48 hours depending on the soiled. At the time of changing the outer gauze a picture of the wound and maggots was provided to individual in charge of insectory and the number of maggots were estimated and adjusted based on the size of infection and recommendations from insectory. After 72 hours all the maggots were removed and the wound was completely cleaned with normal saline. In this particular case, since the patient infection was deep, two additional treatments with maggots, each for three days, were recommended. All the care including the changing of the gauze was performed by the daughter of the patient at home. At the conclusion of maggot therapy, the wound was daily cleaned with sterile solution. A topical antibiotic, Neosporin ointment, was applied to the area for the rest of the treatment time.

\section{Results}

\section{Infection on day 0 of maggot treatment}

As it is shown in Figure $1 \mathrm{~A}$, the size of infection was approximately $3 \times 2$ centimeter, covering the outside of the left feet, right at top of lateral malleolus. Figure 1B shows the Biobag containing larvae on Day 0.

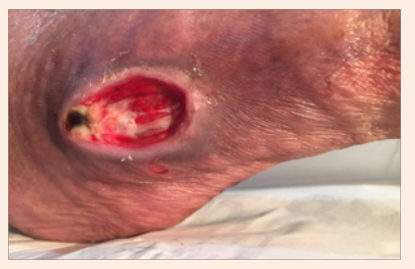

A

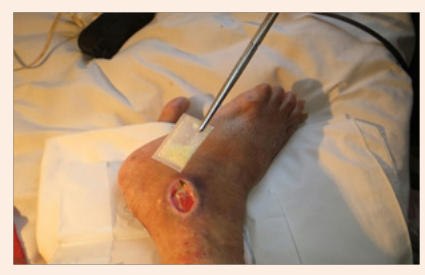

B
Figure 1: Patient's lower foot before maggot therapy.

The wound area was cleaned with normal saline $(1 \mathrm{~A})$ and Biobag containing maggots (1B) was applied to the wound topped with a layer of light gauze.

\section{Infection on day 2 of maggot treatment}

24 hours after the start of treatment, the infection area was examined which indicated $100 \%$ activity of the maggots. Figure $2 \mathrm{~A}$ shows the infection covered by Biobag and Figure 2B reveals the live maggots after removing the Biobag on the same day.

\section{Infection 3 and 5 weeks after maggot treatment}

Wound was checked every day during the changing of the gauze. A significant improvement was observed 3 weeks after maggot therapy and all the infection was eliminated at that point (Figure 3A). The size of the wound was significantly reduced (45\%, Figure 3B) after 5 weeks and new tissue was generated.

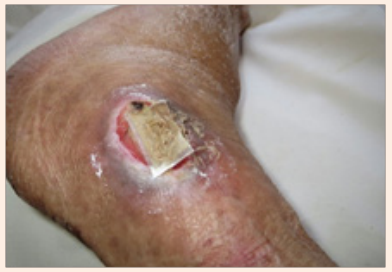

A

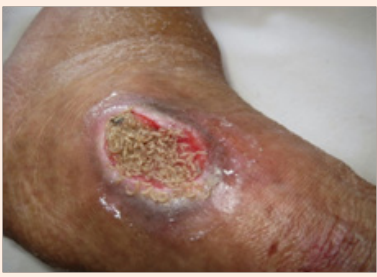

B
Figure 2: Patient's lower foot during maggot therapy.

24 hours after the start of maggot therapy, the infection area was examined. Figure 2A shows the wound area covered by Biobag. Figure $2 \mathrm{~B}$ is reveals the live maggots underneath the Biobag.

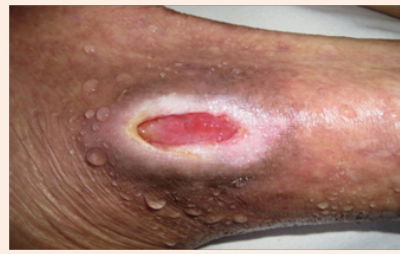

A

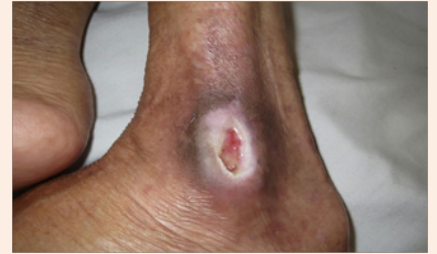

B
Figure 3: Patient's lower foot three and five weeks after maggot therapy.

The size of infection and wound was examined at 3 and 5 weeks after maggot therapy. At 3 weeks, there was no sign of infection (Figure 3A) and the size of the wound was reduced by $45 \%$ at 5 weeks after maggot therapy (Figure 3B).

\section{Infection after three months of initial maggot therapy}

After three months since the start of the maggot therapy, the wound was completely healed and covered with a new layer of skin (Figure 4).

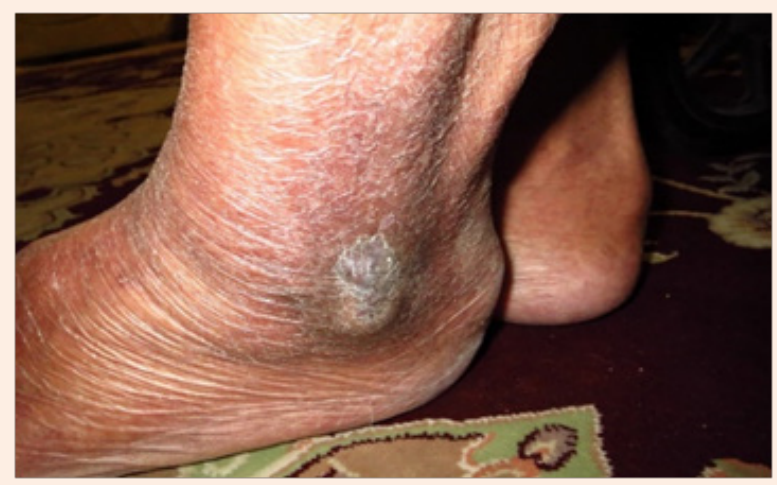

Figure 4: Patient's lower foot three months after maggot therapy.

Examination of the wound three months after maggot therapy revealed a complete healing and closing of the wound. 


\section{Discussion}

The first report of intentional use of maggots in healing of wounds has been reported in the $16^{\text {th }}$ century. The treatment became more popular as more success cases were reported and the first half of the $20^{\text {th }}$ century showed an increased in use of maggots in hospitals. Once antibiotics were introduced, the use of maggots declined but emergence of antibiotic resistance bacteria has again increased the therapeutic use of maggots in wound healing. Reports of successful treatments with maggots in removal of necrotic tissue as well as the increase in tissue repair have been reported by several practitioners [4,5]. In many of these cases, Lucilia sericata is the specie that has shown to be most effective and has been studied in chronic wound healing. Interestingly, in the process of growing, the larvae of $L$. sericata are capable of feeding relatively superficially necrotic tissue without damaging the healthy tissue. Some studies have suggested that maggots contribute to the healing process by removing the necrotic tissues, promoting granulation tissue formation and finally through disinfection of the wound [6,7]. In all three processes, physical digestion of the tissue as well as the secretion of enzymes that aid the processes have been implicated in the removal of infection $[8,9]$. It has been reported that for optimum results, the required number of maggots should be determined using the size and extend of infection. Use of 100 maggots for 50 gram of necrotic tissue or multiplication of the depth of the wound by the surface area have been used as the methods for determination of the number of maggots $[10,11]$.

There reports of the use of maggot therapy in debriding of necrotic tissue, devitalizing tissue and preserving healthy tissue as well as functioning structures has been reported in different cases [12]. Here we have reported successful at-home use of maggots in the healing of an infected wound in an elderly diabetic patient. The patient infection was not responding to antibiotic treatment and at-home therapy with maggot was chosen by patient and his family. Our observation was in agreement with previously reported cases that maggot therapy in combination with topical antibiotics is effective in elimination of infected tissue and regeneration of new tissue which in this case happened within 3 months. It is speculated that the improvement of the healing is the result of physical activity of larvae (by their mandibles) as indicated by accumulation of the maggots mainly on the top of the infection, as well as the secretions and excretions of chemicals such as trypsin, collagenase, and chymotrypsin, which can contribute to the dissolution of necrotic tissue as suggested by other reports [13]. Additionally, it has been reported that several peptides produced in whole body extract of maggots have high antimicrobial activity as well as some compounds present in their haemolymph which have been shown to have activity against P. aeruginosa [4]. Our observations indicate that maggot therapy can be an ideal option in situations where antibiotics are not working especially in elderly patients with difficulty of accessing hospital services. The treatment can be applied by lay person without any complications which save the patient time and money. Additionally, maggot therapy at home may increase the healing process when it is combined with oral antibiotics.

\section{Conclusion}

In conclusion, elderly and low income diabetic patients who have developed antibiotic resistance may benefit from at-home maggot therapy when they develop infections. At home application maggot therapy by a family member is safe, convenient and less expensive as long as the treatment is performed under sterile conditions and at early stages of infection. Diabetic patients without antibiotic resistance may also prefer this method of therapy due to its low cost, convenient and reduced chances of complications for development of antibiotic resistance. At home application of maggot eliminates the inconvenient of transporting the patient to the hospital and can reduce the cost for those who do not have any medical insurance. Still there is a need for larger studies to evaluate the safety and efficacy of maggot therapy and to specify the best conditions under which it can be most effective.

\section{References}

1. http://diabetes.niddk.nih.gov/dm/pubs/statistics/index.htm

2. Wild S, Roglic G, Green A, Sicree R, King H (2004) Global prevalence of diabetes: estimates for the year 2000 and projections for 2030 . Diabetes Care 27(5): 1047-1053.

3. Rafter L (2013) Using larval therapy in the community setting. Wound Care 18(12): S20-S25.

4. Pinheiro MA, Ferraz JB, Junior MA, Moura AD, da Costa ME, et al (2015) Use of maggot therapy for treating a diabetic foot ulcer colonized by multidrug resistant bacteria in Brazil. Indian J Med Res 141(3): 340-342.

5. Gray M (2008) Is larval (maggot) debridement effective for removal of necrotic tissue from chronic wounds? J Wound Ostomy Continence Nurs 35(4): 378-384.

6. Horobin AJ, Shakesheff KM, Pritchard DI (2005) Maggots and wound healing: an investigation of the effects of secretions from Luciliasericatalarvae upon the migration of human dermal fibroblasts over a fibronectin-coated surface. Wound Repair and Regeneration 13(4): 422-433.

7. Church JC (1996) The traditional use of maggots in wound healing, and the development of larva therapy (biosurgeryin modern medicine). J Altern Complement Med 2(4): 525-527.

8. Beasley WD, Hirst G (2004) Making a meal of MRSA - the role of biosurgery in hospital-aquired infection. J Hosp Infect 56(1): 6-9.

9. Murdoch FF and Smart TL (1931) A method of producing sterile blowfly larvae for surgical use. US Navy Bureau of Medicine and Surgery 27: 406-417.

10. Blake FA, Abromeit N, Bubenheim M, Li L, Schmelzle R (2007) The biosurgical wound debridement: experimental investigation of efficiency and practicability. Wound Repair Regen 15(5): 756-761.

11. Lepage OM, Doumbia A, Perron-Lepage MF, Gangl, M (2012) The use of maggot debridement therapy in 41 equids. Equine Veterinary Journal 44 (43): 120-125.

12. Bohac M, Cambal M, Zamborsky R, Takac P, Fedeles J Sr (2015) Maggot therapy in treatment of a complex hand injury complicated by mycotic infection. Bratisl Lek Listy 116(11): 671-673. 
13. Pinheiro MA, Ferraz JB, Junior MA, Moura AD, da Costa ME, et al. (2015). Use of maggot therapy for treating a diabetic foot ulcer colonized by multidrug resistant bacteria in Brazil. Indian J Med Res 141(3): 340-342. 